\title{
Bilateral Endogenous Endophthalmitis Associated With Infective Endocarditis: Case Report
}

\author{
Enyr Saran Arcieri, Eduardo Ferreira Jorge, \\ Lizane de Abreu Ferreira, Maria B. da Fonseca, \\ Magno Antônio Ferreira, Rafael Saran Arcieri \\ and Flávio Jaime Rocha
}

School of Medicine, Federal University of Uberlândia, Uberlândia, MG; Department of Ophthalmology, Glaucoma Service, University of Campinas, SP; Federal University of Mato Grosso do Sul, MS, Brazil

Bilateral endogenous endophthalmitis is a rare condition initiated by infection by microbes in the bloodstream, such as those arising from a foci of infective endocarditis. We report a case and discuss the diagnostic aspects and the clinical outcome of a patient with characteristic findings of the disease. The patient was a 49 year old white male who had a metallic aortic valve implanted 7 months previously, and who presented to the hospital with 10 days of fever, cough and dyspnea, then diarrhea and mental confusion. On the second day of hospitalization, he experienced sudden loss of vision in both eyes. A Gram-positive coccobacillus was isolated from the bloodstream, he was treated with fluoroquinolone with disappearance of fever, decreased ocular inflammation, and improvement in his vision to light perception. He later underwent valve replacement surgery but died during the procedure. We review the occurrence of ocular signs and symptoms and their importance in patients with endocarditis.

Key Words: Bacterial endocarditis, endophthalmitis.

Infectious endophthalmitis is a condition in which the internal structures of the eye become invaded with replicating microorganisms, resulting in an inflammatory response that may ultimately involve all tissues of the eye. Exogenous endophthalmitis occurs when the outer wall of the eye sustains a break due to surgical intervention or trauma [1].

Endogenous endophthalmitis is less common and occurs when the microorganisms spread to the eye from a source elsewhere in the body, usually through the bloodstream [1]. This disease threatens vision and is generally associated with a disease that is also a threat to the patient's life.

Received on 10 October 2001; revised 19 November 2001.

Address for correspondence: Dr. Enyr Saran Arcieri, MD. Rua Corcovado, $n^{\circ} 155$, apto 402 - Bairro Copacabana. Zip Code: 38411-092. Uberlândia - Minas Gerais - Brasil.

E-mail: rsaran@triang.com.br Telephone: (55 34) 3214-8538. Fax:(55 34)3234-2853.

The Brazilian Journal of Infectious Diseases 2001;5(6):356-359. (C) 2001 by The Brazilian Journal of Infectious Diseases and Contexto Publishing. All rights reserved.

1413-8670

\section{Case Report}

A 49-year-old white male, was referred to us with a 10 day history of fever, dry cough and dyspnea. He experienced profuse aqueous diarrhea followed by asthenia and became prostrated 4 days prior to presentation. He also complained of mental confusion 1 day before hospital admission.

The patient's medical history revealed hypertension and double aortic valve lesions. The patient underwent surgery 7 months earlier, to implant a metallic aortic valve prosthesis. The family history was unremarkable. On physical examination, the patient was in regular condition, confused, with respiratory frequency of 24 rpm, pulse frequency of $88 \mathrm{bpm}$, symmetrical with normal amplitudes in the limbs, blood pressure of 150/ $90 \mathrm{mmHg}$, and fever $\left(38.2^{\circ} \mathrm{C} / 101^{\circ} \mathrm{F}\right)$.

The precordium examination showed rhythmic and normophonetic heart sounds, with a holosystolic murmur $1+/ 4+$ in the aortic area and an ejection clicking could be detected in the entire frontal thoracic region. The lungs and abdomen showed no abnormalities on 
physical examination. The ocular examination showed bilateral conjunctival hyperemia and swelling in both upper and lower eyelids.

An echocardiogram (ECO) was requested, a blood culture was performed and the patient was put on $400 \mathrm{mg}$ of fluorquinolone BID. The Grampositive cocobacillus (Streptococcus of Group B) was isolated in the blood culture and the decision was made to maintain the medication.

The diarrhea improved on the second day in the hospital, but the patient complained of sudden loss of vision in both eyes (BE). The visual acuity (VA) decreased to light perception in BE. Biomicroscopy of the right eye (OD) revealed chemosis (edema) and conjunctival hyperemia $2+/ 4+$, transparent cornea and fibrin in the anterior chamber (AC) (Figure 1); the left eye (OS) presented chemosis and conjunctival hyperemia $2+/ 4+$, transparent cornea, posterior synechiae (adhesions between the anterior lens surface and the iris), small quantity of fibrin and haemorrhage in AC (Figure 2). The treatment prescribed in both eyes was fortified cefazolin ${ }^{\circledR}$ eye drops to be used hourly and atropine eye drops to be used every 12 hours.

On the third day, a transesophageal ECO was performed that revealed a normofunctioning and normoimplanted mechanical aortic prosthesis, and regular perivalvar contours that suggested a perivalvar abscess. The isolated agent was identified as Group B streptococcus, resistant only to tetracycline. As the febrile condition persisted, the antibiotic therapy was changed to $60 \mathrm{mg}$ Garamycin, every 8 hours + Penicillin G 3 million U 4/4 h IV.

The patient's systemic and ocular condition improved and the patient no longer had fever. The ocular USG revealed a vitreous cavity replete with non-moveable echoes of medium reflectivity along its entire extension, an image that suggested total detachment of the retina in BE, with very dense subretinal localized echoes with the sac formation in the OD. As the ocular USG image suggested abscess presence in $\mathrm{OD}$, the vitreous was cultured on amikacin and vancomycin was injected inside the vitreous cavity, but no pathogen was isolated from the vitreous culture.
On day 22, the patient presented VA of light perception in the OS and no light perception in the OD. The biomicroscopy revealed total resolution of chemosis and eyelid edema and the inflammatory reaction in both eyes greatly improved.

The patient underwent surgery to change the valvular prosthesis but died during surgery.

\section{Discussion}

Endogenous bacterial endophthalmitis is a rare pathology that affects individuals of any age [2] and represents $2 \%$ to $15 \%$ of all cases of endophthalmitis which, on an average, are only 5 cases for every 10,000 patients hospitalized [3]. The predisposing factors are $[1,2]$ diabetes mellitus, use of intravenous drugs, recent surgery, valvular cardiac diseases, renal insufficiency, neoplasias, corticosteroid therapy, and immunosuppressive therapy. The right eye is generally more affected than the left eye, which is probably due to direct blood flow from the heart [4].

The most common causes of endogenous endophthalmitis are meningitis, endocarditis, urinary tract infection and wound infection [5]. Other sources of infection have included pharyngitis, pneumonia, septic arthritis, pyelonephritis, intra-abdominal abscess and gastrointestinal malignancy. About $25 \%$ of the cases are bilateral and usually caused by [5] meningococcus, Escherichia coli and Klebsiella sp. A review of 72 recent cases, with 18 bilateral cases, revealed 10 cases of endocarditis (13.89\%) [5].

The onset of the signs and symptoms of endophthalmitis depends on the pathogenic virulence. The streptococci infection is generally related to a fulminating process with pain that worsens day by day, chemosis, proptosis, hypopyon, and corneal melting [4].

Blood culture findings are positive in more than $90 \%$ of infective endocarditis cases [2]. With regard to cases of endophthalmitis, a review of 138 cases revealed a positive culture result in $34.8 \%$ of the material aspirated from the anterior chamber, $58.2 \%$ of the material aspirated from vitreous, and $80 \%$ of the vitrectomy material [6]. 
Figure 1. Right Eye. Reveals conjunctival hyperemia and the anterior chamber filled with fibrin

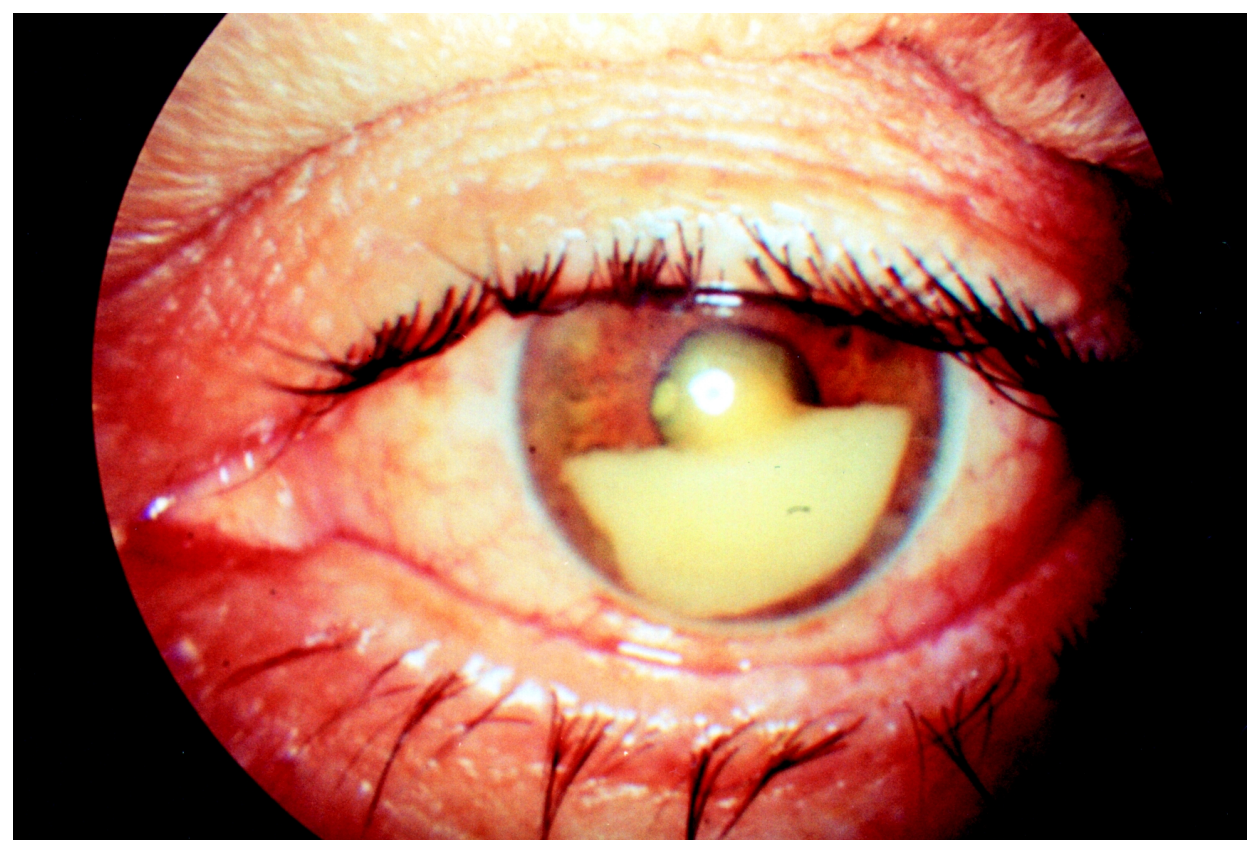

Figure 2. Left Eye. Reveals a small quantity of fibrin and hemorrhagic areas in the anterior chamber

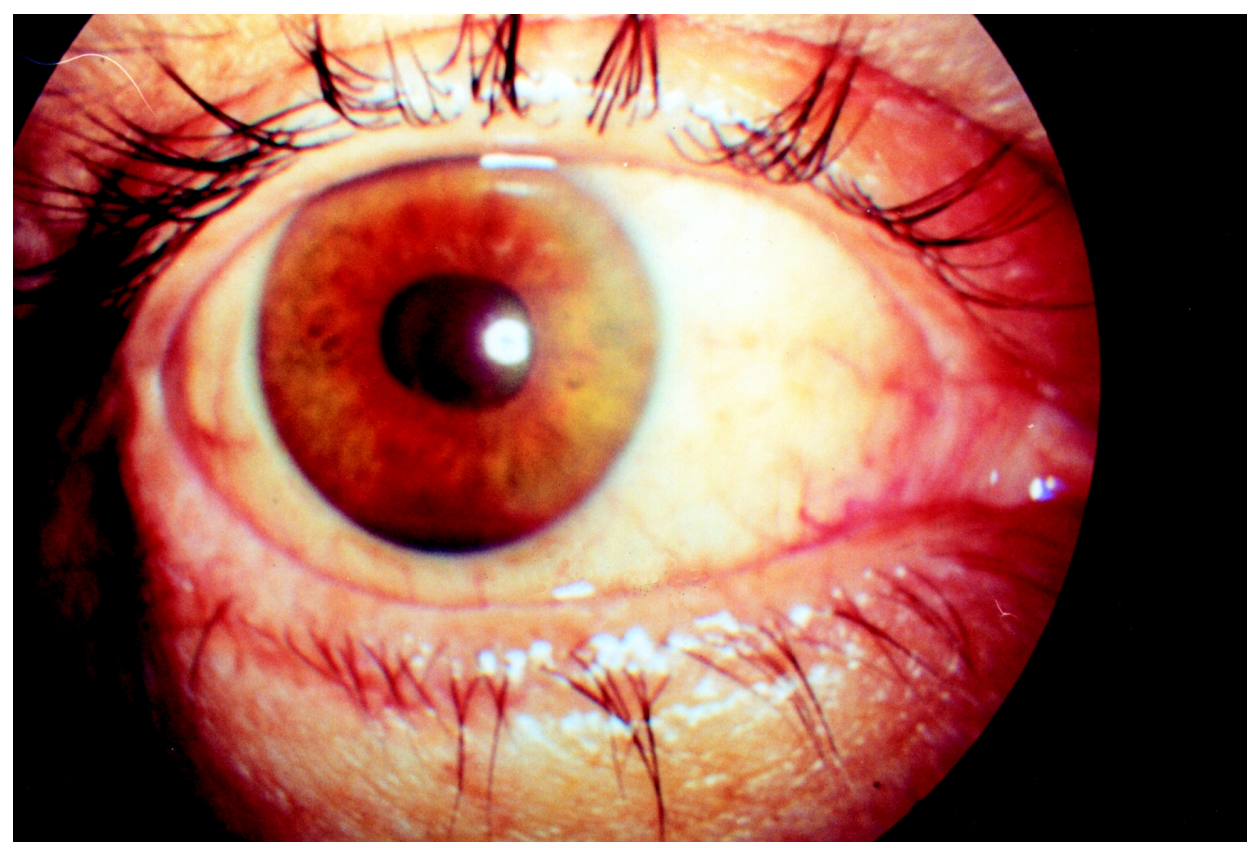


Fabri Jr., et al. [7], conducted a study on infective endocarditis and analyzed 600 episodes with 544 patients. They found that the most common etiological agent was streptococcus (45.7\%), and that the valvular prosthesis was affected in $27.2 \%$ of the episodes.

In a period of 10 years, Okada, et al. [8] reviewed 28 cases that were diagnosed as endogenous endophthalmitis. The most common cause in two-thirds of the infections was Gram-positive bacteria. Although the most common agent was Staphylococcus aureus (25\% of patients), the most common group was streptococcus (32\%). Endogenous endophthalmitis in adults caused by group B Streptococcus has been reported rarely and is almost exclusively related to infective endocarditis [9].

Group B streptococci are facultative Gram-positive diplococci which are usually easily grown. They are universally susceptible to penicillin, ampicillin, vancomycin, first and third generation cephalosporins, and ciprofloxacin [1].

Systemic therapy may be adequate in cases where the vitreous cavity is not greatly involved [4]. Vitrectomy is indicated when microorganisms proliferate in the vitreous cavity, even though intravenous medication could penetrate the invaded site as the inflammatory process increases the power of penetration. Intraocular antibiotic injections are necessary when the eyes do not satisfactorily respond to isolated systemic therapy. Final visual acuity of $20 / 100$ is attained by $30 \%$ of streptococcus infected cases [10]. However, visual prognosis is better related to the patient's initial vision [10], with a marked impairment in presenting visual acuity being related to a poor visual outcome.

Although cardiac complications are the most common complications in patients with infectious endocarditis and can be related to significant mortality and morbidity [7], extra cardiac manifestations generally resulting from thromboembolism, cannot be forgotten. The classic ocular manifestation of infective endocarditis is Roth's spot (hemorrhages with white centers) [2]. Other eye complications that occur are hemorrhagic spots in the conjunctiva and superficial or profound retinal hemorrhage. The occlusion of small arterioles may produce cotton-wool spots or, if the larger vessels are involved, branch or central retinal artery obstruction. Inflammatory complications may occur as a result of septic emboli, and these include acute endophthalmitis, focal abscess, and vasculitis.

\section{References}

1. Meredith T.A. Clinical Microbiology of Infectious Endophthalmitis. In: Ryan S.J., editor. Retina. $2^{\text {nd }}$ ed. St. Louis: Mosby, v.1, chapter16, 1994.

2. Sivalingam A., Bolling J., Goldberg R.E., et al. Ocular abnormalities in acquired heart disease. In: Tasman W., Jaeger E.A., editors. Duane's Clinical Ophthalmology. Philadelphia: JB Lippincott, v.5, chapter 22, 1998.

3. Shrader S.K., Band J.D., Lauter C.B., Murphy P. The clinical spectrum of endophthalmitis: incidence, predisposing factors, and features influencing outcome. J Infect Dis 1990;162(1):115-20.

4. Forster R.K. Endophthalmitis. In: Tasman W., Jaeger E.A., editors. Duane's Clinical Ophthalmology. Philadelphia: JB Lippincott, v.4, chapter 24, 1998.

5. Greenwald M.J., Wohl L.G., Sell C.H. Metastatic bacterial endophthalmitis: A contemporary reappraisal. Surv Ophthalmol 1986;31:81-101.

6. Donahue S.P., Kowalski R.P., Jewart B.H., Friberg T.R. Vitreous cultures in suspected endophthalmitis. Biopsy or vitrectomy?. Ophthalmology 1993;100(4):452-5.

7. Fabri Jr. J., Issa V., Grinberg M., et al. Distribuição temporal das complicações cardíacas em pacientes com endocardite infecciosa. In: Temas Livres do LV Congresso Brasileiro de Cardiologia. Arq Bras Cardiol 2000;74(6):88.

8. Okada A.A., Johnson R.P., Liles W.C., et al. Endogenous bacterial endophthalmitis: Report of a ten-year retrospective study. Ophthalmology 1994;101(5):832-8.

9. Nagelberg H.P., Petashnick D.E., To K.W., Woodcome H.A. Jr. Group B streptococcal metastatic endophthalmitis. Am J Ophthalmol 1994;117(4):498-500.

10. The Endophthalmitis Vitrectomy Study Group. Microbiologic factors and visual outcome in the endophthalmitis vitrectomy study. Am J Ophthalmol 1996;122(6):830-46. 